Pacific Journal of Mathematics

REMARKS ON SIMPLE EXTENDED LIE ALGEBRAS 


\title{
REMARKS ON SIMPLE EXTENDED LIE ALGEBRAS
}

\author{
ARthur A. SAgLe
}

\begin{abstract}
We continue the discussion of finite dimensional simple extended Lie algebras over an algebraically closed field $F$ of characteristic zero with nondegenerate form $(x, y)=$ trace $R_{x} R_{y}$ where $R_{x}$ (or $R(x)$ ) denotes the mapping $A \rightarrow A: a \rightarrow a x$; for brevity we call such an algebra a simple el-algebra. The main result of this paper is that those simple el-algebras which are not Lie or Malcev algebras probably cannot be analyzed by the usual desirable Lie-type methods.
\end{abstract}

First if we assume the simple el-algebra [3] $A$ has a diagonalizable Cartan subalgebra [3] such that for any weight space $A(N, \alpha)$ of $N$ in $A$ we have $A(N, \alpha)^{2}=0$ or $A(N, \alpha)^{2} \subset A(N, \beta)$ for some weight $\beta$ (which is a function of $\alpha$ ), then $A$ is a Lie or Malcev algebra. Thus if one attempts to remedy the situation that $A(N, \alpha)^{2}$ is difficult to locate by the rather desirable above assumptions and tries to construct a multiplication table for a new simple el-algebra, then actually nothing new is obtained. Next we show that if the derivation algebra $D(A)$ is used to analyze a simple el-algebra, using [1, page 54] or possibly Lie module theory, then again a difficult situation is encountered: If $A$ is simple el-algebra, then $A$ is not a simple Lie or Malcev algebra if and only if there exists a nonzero element $a \in A$ such that for every derivation $D \in D(A)$ we have $a D=0$. The element $a \in A$ reflects the structure of $A$ and so it appears that the structure of $A$ is not accurately reflected in its derivation albgebra.

The proofs of the above results use the following lemma.

LEMma 1.1. If $A$ is a simple el-algebra, then $A$ is a Lie or 7-dimensional Malcev algebra if and only if $u(x)=$ trace $R_{x}$ is the zero linear functional.

Proof. A linearization of the defining identities of an extended Lie algebra

$$
x y=-y x \quad \text { and } \quad J(x y, x, y)=0
$$

where $J(x, y, z)=x y \cdot z+y z \cdot x+z x \cdot y$ yields

$$
J(w x, y, z)+J(y z, w, x)=J(w y, z, x)+J(z x, w, y)
$$

Received November 7, 1963. This research was supported in part by NSF Grant GP-1453. 


$$
\begin{aligned}
w J(x, y, z)-x J(y, z, w) & +y J(z, w, x)-z J(w, x, y) \\
& =3[J(w x, y, z)+J(y z, w, x)]
\end{aligned}
$$

for all $w, x, y, z \in A$. From (1.2) we obtain by operating on $w$ that

$$
\begin{aligned}
(x z, y)-(x, z y) & =\operatorname{trace} R(x z) R(y)-\operatorname{trace} R(x) R(z y) \\
& =\operatorname{trace} R(x z \cdot y+x \cdot z y) \\
& =u(x z \cdot y+x \cdot z y) .
\end{aligned}
$$

Now if $u(x)=0$ for all $x \in A$, then from (1.4) we see $(x, y)$ is a nondegenerate invariant form and from [3], $A$ is a simple Lie or 7dimensional Malcev algebra. Conversely, from the identities for these algebras [2] we see that $u(x)=0$ for all $x \in A$.

We continue the use of the notation in [3] for sets and algebraic operations.

2. On the construction. We shall first investigate the assumption that a simple el-algebra $A$ has a diagonalizable Cartan subalgebra $N$ [3]. That is, $N$ is a nilpotent Lie subalgebra of $A$ such that for all $m, n \in N$,

$$
R_{m n}=\left[R_{m}, R_{n}\right] \equiv R_{m} R_{n}-R_{n} R_{m} ;
$$

furthermore, decomposing $A$ into its weight spaces relative to $R(N)=$ $\left\{R_{n}: n \in N\right\}$ we have $[1 ; 3]$

$$
A=A(N, 0) \oplus \sum_{\alpha \neq 0} A(N, \alpha)
$$

where, since $R(N)$ is diagonalizable,

$$
A(N, \lambda)=\left\{x \in A: x R_{n}=\lambda(n) x\right\}
$$

is the weight space of $N$ corresponding to the weight $\lambda$ and, since $N$ is Cartan [3],

$$
N=A(N, 0) \text {. }
$$

Since we are using a fixed Cartan subalgebra we use the notation $A_{\sigma}$ or $A(\sigma)$ for $A(N, \sigma)$ and the convention $A(\sigma)=0$ if $\sigma$ is not a weight of $N$ in $A$. From [3] we have the identities

$$
\begin{aligned}
A_{\alpha} A_{\beta} \subset A_{\alpha+\beta} & \text { if } \alpha \neq \beta \\
J\left(A_{\alpha}, A_{\beta}, A_{\gamma}\right)=0 & \text { if } \alpha \neq \beta \neq \gamma \neq \alpha \\
\text { and } J\left(A_{\alpha}, A_{\beta}, N\right)=0 & \text { if } \alpha \neq \beta .
\end{aligned}
$$

Let $K$ denote the kernel of the linear functional $u: x \rightarrow$ trace $R_{x}$, then we have 


$$
(\alpha+\beta)(n)(x, y)=(\alpha-\beta)(n) u(x y)
$$

$$
\text { if } n \in N, x \in A_{\alpha}, y \in A_{\beta}
$$

For (2.3), let $n \in N$, then $x n=\alpha(n) x, y n=\beta(n) y$ and using (1.4) we have

$$
\begin{aligned}
(\alpha(n)+\beta(n))(x, y) & =(x n, y)-(x, n y) \\
& =u(x n \cdot y+x \cdot n y)=(\alpha(n)-\beta(n)) u(x y) .
\end{aligned}
$$

For (2.4) and (2.5), let $x \in A_{\alpha}, y \in A_{\beta}$ and first assume $\alpha \neq 0$ and $\beta \neq 0$, $\pm \alpha$. If $x y=0$ for all $x, y$ as above, then the results follow from (2.3). So assume $0 \neq x y \in A(\alpha) A(\beta) \subset A(\alpha+\beta)$, then $\alpha+\beta$ is a weight of $N$ in $A$. Let $z \in A(\alpha+\beta)$, then since $\alpha \neq \alpha+\beta \neq \beta \neq \alpha$ we use (2.2) to obtain $J(x, y, z) \in J(A(\alpha), A(\beta), A(\alpha+\beta))=0$. Therefore

$$
\begin{aligned}
z R(x y)=z x \cdot y & +y z \cdot x \in A(2 \alpha+\beta) A(\beta) \\
& +A(\alpha+2 \beta) A(\alpha) \subset A(2(\alpha+\beta)) .
\end{aligned}
$$

Using this result and (2.1) we see that for any weight $\gamma$,

$$
A(\gamma) R(x y) \subset A(\gamma+(\alpha+\beta)) \neq A(\gamma)
$$

and therefore the matrix for $R(x y)$ has zeros on its diagonal so that $u(x y)=$ trace $R(x y)=0$. Next we relax the assumptions on $\beta$, use the above result and (2.3) to see that (2.4) and (2.5) now follow.

Now we shall start using the hypothesis that if $\alpha$ is any weight of $N$ in $A$, then $A_{\alpha}^{2}=0$ or there exists a weight $\pi(\alpha)$ such that $A_{a}^{2} \subset A_{\pi(\alpha)}$. Thus we are assuming that if $A_{\alpha}^{2} \neq 0$, then there exists a weight $\pi(\alpha)$ such that for each $x, y \in A_{\alpha}, x y \in A_{\pi(\alpha)}$; that is, $\pi$ is a function of the weight and not a function of the particular elements used in forming the products. Using this assumption we shall show that for any weight $\alpha, A_{\alpha} \subset K$ (= kernel of $u$ ) and therefore by Lemma 1.1 conclude that $A$ is Lie or Malcev.

First for $\alpha=0$ we have $A_{0}^{2}=A_{0} N=0$. So assume $\alpha \neq 0$. If $x y=0$ for all $x, y \in A_{\alpha}$, then using (2.1) we see that for any $x \in A_{\alpha}$, $u(x)=\operatorname{trace} R_{x}=0$ and therefore $A_{\alpha} \subset K$. So next we consider $0 \neq$ $A_{\alpha}^{2} \subset A_{\pi(\alpha)}$ where $\alpha \neq 0$.

Lemma 2.6. If $\alpha \neq 0$ and $0 \neq A_{\alpha}^{2} \subset A_{\pi(\alpha)}$, then $\pi(\alpha) \neq 0$.

Corollary 2.7. $N=\sum_{\alpha \neq 0} A(\alpha) A(-\alpha) \subset K$. 
Suppose Lemma 2.6 has been proven, then to prove the corollary we first note $\sum_{\alpha \neq 0} A(\alpha) A(-\alpha) \subset A(0)=N$. Next set $B=\sum_{\alpha \neq 0} A(\alpha)(-\alpha) \oplus$ $\sum_{\alpha \neq 0} A(\alpha)$; we shall show $B$ is an ideal of $A$. For any weight $\beta \neq 0$,

$$
\begin{aligned}
B A(\beta) \subset\left(\sum_{\alpha \neq 0} A(\alpha) A(-\alpha)\right) A(\beta)+A(\beta)^{2} & \\
& +A(\beta) A(-\beta)+\sum_{\alpha \neq 0, \pm \beta} A(\alpha+\beta) .
\end{aligned}
$$

Then using $A(\beta)^{2}=0$ or $A(\beta)^{2} \subset A(\pi(\beta))$, where from Lemma 2.6. $\pi(\beta) \neq 0$, we see that $B A(\beta) \subset B$. For $\beta=0$ we note that

$$
\left(\sum_{\alpha \neq 0} A(\alpha) A(-\alpha)\right) A(0) \subset A(0) N=0
$$

and use (2.1) to obtain $B A(0) \subset B$. Thus $B A \subset B$ so that $B$ is an ideal of $A$ and since $A$ is simple, $B=0$ or $B=A$. If $B=0$, then $A_{\alpha}=0$ for each $\alpha \neq 0$ and $A=A_{0}=N$ so that $A^{2}=A_{0} N=0$, a contradiction. Thus $B=A$ and from this $N=\sum_{\alpha \neq 0} A(\alpha) A(-\alpha) \subset K$, using: (2.5).

For Lemma 2.6 assume $\pi(\alpha)=0$ and let $x, y \in A_{\alpha}$, then $x y \in A_{\alpha}^{2} \subset$ $A_{0}=N$. We shall show for any weight $\beta$ that $\beta(x y)=0$, then for any $z \in A_{\beta}$ we have $z(x y)=z R(x y)=\beta(x y) z=0$. Therefore $(x y) F$ is an ideal of $A$ which must be zero and so $A_{\alpha}^{2}=0$, a contradiction. For $x, y \in A_{\alpha}$ we have from the defining identity

$$
0=J(x y, x, y)=(x y \cdot x) y+(y \cdot x y) x
$$

which implies, since $x y \in N, 2 \alpha(x y) x y=0$. From this and the fact that $\alpha$ is a linear functional on $N$ we have $2 \alpha(x y)^{2}=0$ and so $\alpha(x y)=0$. Thus for $\beta=0, \alpha$ we have $\beta(x y)=0$ so we now assume $\beta \neq 0, \alpha$ and let $z \in A_{\beta}, n \in N$, then using (2.1) and (2.2) we obtain

$$
\begin{aligned}
J(z x, y, n)+J(y n, z, x) & =\alpha(n) J(y, z, x) \\
& =-\alpha(n) \beta(x y) z+\alpha(n)(y z \cdot x+z x \cdot y)
\end{aligned}
$$

and

$$
\begin{aligned}
J(z n, x, y)+J(x y, z, n) & =\beta(n) J(z, x, y) \\
& =-\beta(n) \beta(x y) z+\beta(n)(y z \cdot x+z x \cdot y) .
\end{aligned}
$$

We combine these equations by using (1.2) to obtain

$$
\alpha(n)(-\beta(x y) z+z x \cdot y+y z \cdot x)=\beta(n)(-\beta(x y) z+z x \cdot y+y z \cdot x) .
$$

From this equality we obtain, since $\beta(n) \neq \alpha(n)$ for some $n$, that

$$
\beta(x y) z=z x \cdot y+y z \cdot x \in A(2 \alpha+\beta) .
$$

But since $\beta(x y) z \in A(\beta)$ we have

$$
\beta(x y) z \in A(\beta) \cap A(2 \alpha+\beta)=0 .
$$


Thus if $z \neq 0, \beta(x y)=0$ and this proves the lemma.

Thus far we have considered for $\alpha \neq 0$ : (1) $A_{\alpha}^{2}=0$ which implies $A_{\alpha} \subset K$; (2) $A_{\alpha}^{2} \neq 0$ which implies $\pi(\alpha) \neq 0$ and consequently $N=A_{0}=$ $\sum_{\alpha \neq 0} A(\alpha) A(-\alpha) \subset K$. So we next investigate (2) more closely and note that it suffices to consider $0 \neq A_{\alpha}^{2} \subset A_{\pi(\alpha)}$ where $\pi(\alpha)=\alpha$. For if $\pi(\alpha) \neq \alpha$, then using (2.1) we see that the matrix of $R_{x}$ for any $x \in A_{\alpha}$ has zeros on its diagonal and therefore $u(x)=0$ so that $A_{\alpha} \subset K$ which is what we eventually want to show for any weight $\alpha$.

Thus we are considering $0 \neq A_{\alpha}^{2} \subset A_{\alpha}$. Since $(x, y)$ is nondegenerate and $A_{\alpha}^{2} \neq 0$, there exists a weight $\beta$ so that

$$
\left(A_{\alpha}^{2}, A_{\beta}\right) \neq 0 \text {. }
$$

But since $A_{\alpha}^{2} \subset A_{\alpha}$ this means $\left(A_{\alpha}, A_{\beta}\right) \neq 0$ and from (2.4) and the assumption that $\alpha \neq 0$ we conclude $\beta=0$ or $\beta=-\alpha$. We shall consider these two cases and show that the situation $0 \neq A_{\alpha}^{2} \subset A_{\alpha}$ actually does not exist so that we may conclude that for any weight $\alpha, A_{\alpha} \subset K$.

Case $\beta=0$. Let $x, y \in A_{\alpha}, n \in A_{0}$ and $x y \in A_{\alpha}$, then using $\left(A_{\alpha}, A_{\alpha}\right)=$ 0 (from (2.3)) we have

$$
\begin{aligned}
(x y, n) & =(x y, n)-(x, y n) \\
& =u(x y \cdot n+x \cdot y n) \\
& =u(\alpha(n) x y+\alpha(n) x y) \\
& =2 \alpha(n) u(x y) .
\end{aligned}
$$

However from (2.3) and $x y \in A_{\alpha}$ we have

$$
\begin{aligned}
\alpha(n)(x y, n) & =(\alpha+0)(n)(x y, n) \\
& =(\alpha-0)(n) u(x y \cdot n) \\
& =\alpha(n)^{2} u(x y) .
\end{aligned}
$$

From (2.8) we also have $\alpha(n)(x y, n)=2 \alpha(n)^{2} u(x y)$ and therefore from

$$
\alpha(n)^{2} u(x y)=0 \quad \text { for all } n \in N, x, y \in A_{\alpha} .
$$

Now there exists $x, y \in A_{\alpha}$ so that $u(x y) \neq 0$, otherwise from (2.8) we would have $\left(A_{\alpha}^{2}, A_{0}\right)=0$, contrary to our assumption for case $\beta=0$. But from the previous equation this implies $\alpha(n)=0$ for all $n \in N$, contradicting the assumption $\alpha \neq 0$. Thus case $\beta=0$ does not exist.

Case $\beta=-\alpha$. That is, $\alpha \neq 0, A_{\alpha}^{2} \subset A_{\alpha}$ and $\left(A_{\alpha}^{2}, A_{\beta}\right) \neq 0$ with $\beta=-\alpha$; in particular we are assuming $-\alpha$ is a weight. We shall show in this case that the dimension of $A_{\alpha}$ is one and therefore $A_{\alpha}^{2}=0$, a contradiction; thus case $\beta=-\alpha$ does not exist. So assume the dimension of $A_{\alpha}$ is greater than one and let $x, y \in A_{\alpha}, z \in A_{-\infty}$ and $n \in N$, then using $x y \in A_{\alpha}$ and (2.2) we have

$$
J(n y, z, x)+J(z x, n, y)=-\alpha(n) J(y, z, x)
$$


and

$$
J(n z, x, y)+J(x y, n, z)=\alpha(n) J(z, x, y) .
$$

Applying (1.2) to these equations we have, since $\alpha \neq 0$,

$$
\begin{aligned}
0=J(y, z, x) & =y z \cdot x+z x \cdot y+x y \cdot z \\
& =x y \cdot z-\alpha(y z) x-\alpha(z x) y .
\end{aligned}
$$

Therefore since $x y \cdot z \in A_{0}$ and $x, y \in A_{\alpha}$ we have $x y \cdot z=0$ and $\alpha(y z) x+$ $\alpha(z x) y=0$. But since we have assumed the dimension of $A_{\alpha}>1$ and $x, y$ are arbitrary in $A_{\alpha}$ we have $\alpha(z x)=0$ for any $z \in A_{-\alpha}$; for just choose $0 \neq x$ arbitrary in $A_{\alpha}$ and $y$ to be linearly independent of $x$, then for any $z \in A, \alpha(y z) x+\alpha(z x) y=0$ which yields the result.

Next we shall show $\beta(z x)=0$ for any weight $\beta$ of $N$ and any $z \in A(-\alpha), x \in A(\alpha)$. If $\beta=q \alpha$ where $q$ is a rational number, the results follow. Next suppose $\beta \neq q \alpha$ and let $M=\sum_{k} A(\beta+k \alpha), k=$ $0, \pm 1, \pm 2, \cdots$. Using (2.1) and $\beta \neq q \alpha$ we see that $M$ is $R_{x}$-, $R_{z}-$, and $R(x z)$-invariant and for any $y=\sum_{k} y_{k} \in M$ where $y_{k} \in A(\beta+k \alpha)$ we have

$$
J(y, x, z)=\sum_{k} J\left(y_{k}, x, z\right)=0,
$$

using (2.2). Thus $y\left(\left[R_{x}, R_{z}\right]-R(x z)\right)=0$; that is, on $M$ we have $R(x z)=\left[R_{x}, R_{z}\right]$ so that

$$
\operatorname{trace}_{M} R(x z)=0,
$$

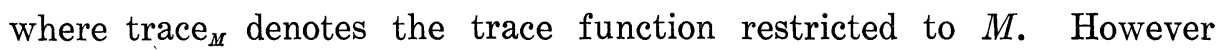
calculating the trace ${ }_{M} R(x z)$ from the matrix of $R(x z)$ on $M$ we see that

$$
\begin{aligned}
\operatorname{trace}_{M} R(x z) & =\sum_{k} N_{k}(\beta+k \alpha)(x z), \quad N_{k}=\operatorname{dim} A(\beta+k \alpha) \\
& =\left(\sum_{k} N_{k}\right) \beta(x z)+\left(\sum_{h} k N_{k}\right) \alpha(x z) \\
& -\left(\sum_{k} N_{k}\right) \beta(x z), \text { since } \alpha(x z)=0 .
\end{aligned}
$$

This equation and (2.10) imply $\beta(x z)=0$. Thus for any weight $\beta$ and any $y \in A_{\beta}$ we have $y R(x z)=\beta(x z) y=0$ which implies $R(x z)=0$ and therefore $x z=0$ i.e. $A(\alpha) A(-\alpha)=0$. We use this fact to obtain a contradiction to $\left(A^{2}(\alpha), A(-\alpha)\right) \neq 0$. So let $x, y \in A(\alpha), z \in A(-\alpha)$, then using (1.4) we have

$$
\begin{aligned}
(x y, z) & =(x, y z)+u(x y \cdot z+x \cdot y z) \\
& =u(x y \cdot z), \text { using } y z \in A(\alpha) A(-\alpha)=0 \\
& =0, \text { using } x y \in A(\alpha) \text { and } A(\alpha) A(-\alpha)=0 .
\end{aligned}
$$

This contradiction shows case $\beta=-\alpha$ does not exist and so from previous remarks we have for any weight $\alpha, A_{\alpha} \subset K$ which proves 
following conditions

(1) there exists a Cartan subalgebra $N$ of $A$ so that $R(N)=$ $\left\{R_{n}: n \in N\right\}$ acts diagonally in $A$

(2) if $A=\sum_{\alpha} A(N, \alpha)$ is the weight space decomposition of $A$ relative to $R(N)$ where $N$ is the subalgebra of (1), then $A(N, \alpha)^{2}=0$ or $A(N, \alpha)^{2} \subset A(N, \pi(\alpha))$ for some weight $\pi(\alpha)$.

Then $A$ is a Lie or 7-dimensional Malcev algebra.

3. On derivations. Again let $A$ be a simple el-algebra. To use the derivation algebra $D(A)$ in the analysis of $A$ we first locate the derivations of $A$ as follows.

THeOREM 3.1. Every derivation of $A$ is inner, that is, $D(A)$ is contained in the Lie trasformation algebra $L(A)$ which is the smallest Lie algebra containing $R(A)=\left\{R_{x}: x \in A\right\}$ [4].

Proof. Since $A$ is simple it contains no nontrivial $L(A)$-invariant subspaces and so $L(A)$ is irreducible in $A$. This implies $L(A)=C \oplus$ $L(A)^{\prime}$ where $C$ is the center of $L(A)$ and $L(A)^{\prime}=[L(A), L(A)]$ is semisimple [1; Th. 2.11]. Furthermore $C=0$ or $C=F I$; for if $S$ is a linear transformation in $C$, then since $F$ is algebraically closed $S$ has a characteristic root $\lambda$ in $F$. Using the fact $[R(A), S]=0$ we see $\{x \in A: x S=\lambda x\}$ is a nonzero ideal of $A$ and therefore equals $A$. From this the results concerning $C$ follow.

Now let $D \in D(A)$, then we have $\left[R_{x}, D\right]=R(x D)$ for all $x \in A$ and this together with the Jacobi identity imply $\left[L(A)^{\prime}, D\right] \subset L(A)^{\prime}$. Thus the mapping

$$
L(A)^{\prime} \rightarrow L(A)^{\prime}: X^{\prime} \rightarrow\left[X^{\prime}, D\right] \quad \text { all } X^{\prime} \in L[A]^{\prime}
$$

is a derivation of $L(A)^{\prime}$. Since $L(A)^{\prime}$ is semi-simple every derivation of $L(A)^{\prime}$ is inner and therefore there exists $D^{\prime} \in L(A)^{\prime}$ so that $\left[X^{\prime}, D\right]=$ $\left[X^{\prime}, D^{\prime}\right]$ all $X^{\prime} \in L(A)^{\prime}\left[1\right.$; Th. 3.6]. But for any $X=a I+X^{\prime} \in L(A)$ where $a \in F$ (if $C \neq 0$ ) we have $[X, D]=\left[X, D^{\prime}\right]$. Thus if $T=D-D^{\prime}$ we have in particular that $[R(A), T]=0$. Again since $F$ is algebraically closed $T$ has a characteristic root $\mu$ and we see that $\{x \in A: x T=$ $\mu x\}$ is a nonzero ideal in $A$. This implies either $T=0$ in which case $D=D^{\prime}$ or $T=\mu I$ in which case $D=\mu I+D^{\prime}$. Now in this latter case we note $D^{\prime} \in L(A)^{\prime}$ so that trace $D^{\prime}=0$ and since $(x, y)=$ trace $R_{x} R_{y}$ is nondegenerate we have from $\left[R_{x}, D\right]=R(x D)$ that $(x D, y)+$ $(x, y D)=0$ so that $D$ is skewsymmetric and also trace $D=0$. From these facts on trace and $D=\mu I+D^{\prime}$ we conclude $D=D^{\prime} \in L(A)$ in both cases.

Even though we know all derivations of a simple el-algebra are inner, their exact form has not yet been determined. However the 
following is not too difficult to prove: If $A$ is a simple el-algebra, then $A$ is a Lie algebra if and only if there exists an element $x \in A$ so that $R_{x}$ is a nonzero derivation of $A$. Next we have

Theorem 3.2. If $A$ is a simple el-algebra, then $A$ is not a Lie or 7-dimensional Malcev algebra if and only if there exists a nonzero element $a \in A$ such that for every derivation $D$ of $A$ we have $a D=0$.

Proof. If $A$ is a Lie or 7-dimensional Malcev algebra then the conclusion is well known [2]. Conversely, if $A$ is not Lie or 7-dimensional Malcev, then since $(x, y)=$ trace $R_{x} R_{y}$ is nondegenerate we use Lemma 1.1 to obtain a nonzero element $a \in A$ so that for all $x \in A$, $u(x)=(x, a)$. But for any derivation $D$ we have $R(x D)=\left[R_{x}, D\right]$ and $(x D, y)+(x, y D)=0$ so that in particular we have for any $x \in A$, $(a D, x)=-(a, x D)=-u(x D)=-\operatorname{trace} R(x D)=0$. Thus since $(x, y)$ is nondegenerate $a D=0$.

\section{REFERENCES}

1. N. Jacobson, Lie algebras, Interscience, 1962.

2. A. Sagle, Malcev algebras, Trans. Amer. Math. Soc. 101 (1961), 426-458.

3. __ On simple extended Lie algebras over fields of characteristic zero, to appear.

4. R.D. Schafer, Inner derivations of nonassociative algebras, Bull. Amer. Math. Soc. 55 (1949), 769-776.

UNIVERSiTy OF CALIFORNiA, LOS ANGELES 


\title{
PACIFIC JOURNAL OF MATHEMATICS
}

\author{
EDITORS
}

\author{
H. Samelson \\ Stanford University \\ Stanford, California \\ R. M. Blumenthal \\ University of Washington \\ Seattle, Washington 98105
}

\author{
J. DugundjI \\ University of Southern California \\ Los Angeles, California 90007 \\ Richard Arens \\ University of California \\ Los Angeles, California 90024
}

\section{ASSOCIATE EDITORS}
E. F. BECKENBACH
B. H. NEUMaNN
F. WOLF
K. YosIDA

\section{SUPPORTING INSTITUTIONS}

\author{
UNIVERSITY OF BRITISH COLUMBIA \\ CALIFORNIA INSTITUTE OF TECHNOLOGY \\ UNIVERSITY OF CALIFORNIA \\ MONTANA STATE UNIVERSITY \\ UNIVERSITY OF NEVADA \\ NEW MEXICO STATE UNIVERSITY \\ OREGON STATE UNIVERSITY \\ UNIVERSITY OF OREGON \\ OSAKA UNIVERSITY \\ UNIVERSITY OF SOUTHERN CALIFORNIA
}

\author{
STANFORD UNIVERSITY \\ UNIVERSITY OF TOKYO \\ UNIVERSITY OF UTAH \\ WASHINGTON STATE UNIVERSITY \\ UNIVERSITY OF WASHINGTON \\ AMERICAN MATHEMATICAL SOCIETY \\ CALIFORNIA RESEARCH CORPORATION \\ SPACE TECHNOLOGY LABORATORIES \\ NAVAL ORDNANCE TEST STATION
}

Mathematical papers intended for publication in the Pacific Journal of Mathematics should by typewritten (double spaced). The first paragraph or two must be capable of being used separately as a synopsis of the entire paper. It should not contain references to the bibliography. Manuscripts may be sent to any one of the four editors. All other communications to the editors should be addressed to the managing editor, Richard Arens, at the University of California, Los Angeles, California 90024.

50 reprints per author of each article are furnished free of charge; additional copies may be obtained at cost in multiples of 50 .

The Pacific Journal of Mathematics is published quarterly, in March, June, September, and December. Effective with Volume 13 the price per volume (4 numbers) is $\$ 18.00$; single issues, $\$ 5.00$. Special price for current issues to individual faculty members of supporting institutions and to individual members of the American Mathematical Society: $\$ 8.00$ per volume; single issues $\$ 2.50$. Back numbers are available.

Subscriptions, orders for back numbers, and changes of address should be sent to Pacific Journal of Mathematics, 103 Highland Boulevard, Berkeley 8, California.

Printed at Kokusai Bunken Insatsusha (International Academic Printing Co., Ltd.), No. 6, 2-chome, Fujimi-cho, Chiyoda-ku, Tokyo, Japan.

PUBLISHED BY PACIFIC JOURNAL OF MATHEMATICS, A NON-PROFIT CORPORATION

The Supporting Institutions listed above contribute to the cost of publication of this Journal, but they are not owners or publishers and have no responsibility for its content or policies. 


\section{Pacific Journal of Mathematics}

\section{Vol. 15, No. $2 \quad$ October, 1965}

Patrick Robert Ahern, On the generalized F. and M. Riesz theorem......... 373

A. A. Albert, On exceptional Jordan division algebras ................ 377

J. A. Anderson and G. H. Fullerton, On a class of Cauchy exponential

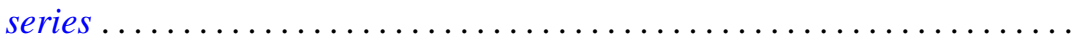

Allan Clark, Hopf algebras over Dedekind domains and torsion in

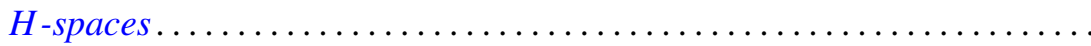

John Dauns and D. V. Widder, Convolution transforms whose inversion functions have complex roots .............................

Ronald George Douglas, Contractive projections on an $\mathrm{L}_{1}$ space ..........

Robert E. Edwards, Changing signs of Fourier coefficients ...............

Ramesh Anand Gangolli, Sample functions of certain differential processes on symmetric spaces .....................................

Robert William Gilmer, Jr., Some containment relations between classes of

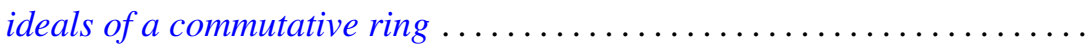

Basil Gordon, A generalization of the coset decomposition of a finite

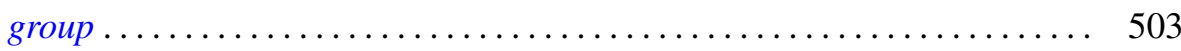

Teruo Ikebe, On the phase-shift formula for the scattering operator....... 511

Makoto Ishida, On algebraic homogeneous spaces ................ 525

Donald William Kahn, Maps which induce the zero map on homotopy ........ 537

Frank James Kosier, Certain algebras of degree one ................. 541

Betty Kvarda, An inequality for the number of elements in a sum of two sets of lattice points.................................

Jonah Mann and Donald J. Newman, The generalized Gibbs phenomenon for regular Hausdorff means. .

Charles Alan McCarthy, The nilpotent part of a spectral operator. II . ...

Donald Steven Passman, Isomorphic groups and group rings ...

R. N. Pederson, Laplace's method for two parameters .....

Tom Stephen Pitcher, A more general property than domination for sets of probability measures .............................

Arthur Argyle Sagle, Remarks on simple extended Lie algebras. .

Arthur Argyle Sagle, On simple extended Lie algebras over fields of

characteristic zero.

Tôru Saitô, Proper ordered inverse semigroups ...........

Oved Shisha, Monotone approximation

Indranand Sinha, Reduction of sets of matrices to a triangular form

Raymond Earl Smithson, Some general properties of multi-valued

functions .................................

John Stuelpnagel, Euclidean fiberings of solvmanifolds .... 